\title{
HIGH ENERGY FACTORIZATION AT NLO: LIPATOV'S EFFECTIVE ACTION REVISITED*
}

\author{
G. CHACHAMIS \\ Instituto de Física Corpuscular UVEG/CSIC, Valencia, Spain \\ grigorios.chachamis@ific.uv.es \\ M. HENTSCHINSKI ${ }^{\dagger}$ \\ Physics Department, Brookhaven National Laboratory, Upton, NY, USA \\ hentsch@bnl.gov \\ J. D. MADRIGAL MARTÍNEZ \\ Instituto de Física Teórica UAM/CSIC \\ Universidad Autónoma de Madrid, Madrid, Spain \\ josedaniel.madrigal@uam.es \\ A. SABIO VERA \\ Instituto de Física Teórica UAM/CSIC \\ Universidad Autónoma de Madrid, Madrid, Spain \\ agustin.sabio@uam.es
}

Published 2 January 2014

\begin{abstract}
We discuss aspects of our recent derivation of the gluon Regge trajectory at two loop from Lipatov's high energy effective action. We show how the gluon Regge trajectory can be rigorously defined through renormalization of the high energy divergence of the reggeized gluon propagator. We furthermore provide some details on the determination of the two-loop reggeized gluon self-energy.
\end{abstract}

Keywords: perturbative QCD; high energy factorization; effective field theories.

\section{Introduction}

Balitsky-Fadin-Kuraev-Lipatov (BFKL) resummation ${ }^{1,2}$ and high energy factorization provide the basis for the study of the high energy limit of hard QCD scattering processes. Recent phenomenological evidence for BFKL evolution is found

*This is an Open Access article published by World Scientific Publishing Company. It is distributed under the terms of the Creative Commons Attribution 3.0 (CC-BY) License. Further distribution of this work is permitted, provided the original work is properly cited.

†Speaker 
in the analysis of the combined HERA data on the structure function $F_{2}$ and $F_{L}$ $[3,4]$ and the study of di-hadron spectra in high multiplicity distributions at the Large Hadron Collider. ${ }^{5}$ An interesting application to phenomenology is furthermore provided by Transverse-Momentum-Dependent parton distribution functions, especially in region of small parton momentum fraction $x$, where high energy factorization provides a natural definition, see Ref. [6] for recent work.

An attractive approach for the theoretical investigation of high energy factorization is provided by Lipatov's gauge invariant high energy effective action ${ }^{7}$ which provides a re-formulation of QCD at high center of mass energies as an effective field theory of reggeized gluons. In Ref. [9] a scheme has been developed which comprises regularization, subtraction and renormalization of high energy divergences and allows to use the high energy effective action for the determination of nextto-leading order corrections to high energy factorized matrix elements. So far this scheme has been successfully applied to the derivation of forward jet vertices for both quark ${ }^{9}$ and gluon ${ }^{10}$ initiated jets at NLO accuracy.

In Ref. $[11,12]$ this programme has been extended to the calculation of the 2loop gluon Regge trajectory. This universal function associated with the exchange of a single reggeized gluon provides a key ingredient in the formulation of high energy factorization and the resummation of high energy logarithms of QCD scattering amplitudes. Multiple reggeized gluon exchanges appear on the other hand for the high energy description of the imaginary part of scattering amplitudes and in general for amplitudes beyond NLL accuracy. While this requires new elements, which describe in a nutshell the interaction between reggeized gluons, the gluon Regge trajectory remains an essential building block in the formulation of high energy resummation also in this more general case. To be more precise, for the elastic process $p_{a}+p_{b} \rightarrow p_{1}+p_{2}$ with $s=\left(p_{a}+p_{b}\right)^{2}$ and $t=q^{2}$ with $q=p_{a}-p_{1}$ one finds for amplitudes with gluon quantum numbers in the $t$-channel at LL and NLL accuracy the following factorized form ${ }^{\mathrm{a}}$

$$
\frac{\mathcal{M}_{\left(\mathbf{8}_{\mathbf{A}}\right)}(s, t)}{\mathcal{M}^{(0)}(s, t)}=\Gamma_{a 1}(t)\left[\left(\frac{-s}{-t}\right)^{\omega(t)}+\left(\frac{s}{-t}\right)^{\omega(t)}\right] \Gamma_{b 2}(t),
$$

where $\mathcal{M}_{\left(\mathbf{8}_{\mathbf{A}}\right)}^{(0)}$ is the tree-level amplitude and the subscript ' $\mathbf{8}_{\mathbf{A}}$ ' denotes that the allowed $t$-channel exchange is restricted to the anti-symmetric color octet channel. The functions $\Gamma_{i j}(t)$ are known as impact factors, describing the coupling of the reggeized gluons to scattering particles. For the case of gluon and quarks they have been determined within the effective action in Ref. $[9,10]$. The function $\omega(t)$ which governs the $s$-dependence of the scattering amplitude is on the other hand the Regge trajectory of the gluon. It has been originally derived in Ref. [13, 14] using $s$ channel unitarity relations. The result was then subsequently confirmed in Ref. [15], clarifying an ambiguity in the non-infrared divergent contributions of Ref. [16].

${ }^{a}$ For a pedagogical review see Ref. [20]. 
The original result was further verified by explicitly evaluating the high energy limit of 2-loop partonic scattering amplitudes Ref. [17]. While the explicit result for the 2-loop gluon Regge trajectory is by now firmly established, our calculation provides an important confirmation of its universality: unlike previous calculations, the effective action defines the Regge trajectory of the gluon without making any reference to a particular QCD scattering process.

The outline of this contribution is as follows: Sec. 2 provides a short introduction to Lipatov's effective action and a list of necessary Feynman rules, together with a discussion of our regularization and the employed pole prescription. In Sec. 3 we recall the scheme which we follow in the derivation of the gluon Regge trajectory and presents our result. Sec. 4 contains our conclusions.

\section{Lipatov's High Energy Effective Action}

The effective action ${ }^{7}$ describes interactions which are restricted to an interval of narrow width $(\eta)$ in rapidity space. Dynamics extending over rapidity separations larger than $\eta$, is integrated out and taken into account through universal eikonal factors. QCD amplitudes are then in the limit of large center of mass energies constructed through a new degree of freedom - the reggeized gluon. The high energy effective action describes the interaction of this new field with the QCD field content through adding an induced term $S_{\text {ind. }}$ to the QCD action $S_{\mathrm{QCD}}$,

$$
S_{\mathrm{eff}}=S_{\mathrm{QCD}}+S_{\mathrm{ind}} \cdot
$$

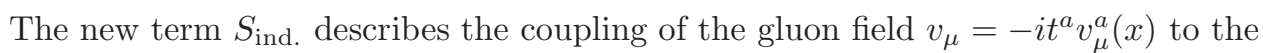
reggeized gluon field $A_{ \pm}(x)=-i t^{a} A_{ \pm}^{a}(x)$. High energy factorized amplitudes reveal strong ordering in plus and minus components of momenta which is reflected in the following kinematic constraint obeyed by the reggeized gluon field

$$
\partial_{+} A_{-}(x)=0=\partial_{+} A_{+}(x) .
$$

Even though the reggeized gluon field is charged under the QCD gauge group $\mathrm{SU}\left(N_{c}\right)$, it is invariant under local gauge transformations: $\delta A_{ \pm}=0$. Its kinetic term and the gauge invariant coupling to the QCD gluon field are contained in the induced term,

$$
S_{\text {ind. }}=\int \mathrm{d}^{4} x \operatorname{tr}\left[\left(W_{-}[v(x)]-A_{-}(x)\right) \partial_{\perp}^{2} A_{+}(x)\right]+(+) \leftrightarrow(-),
$$

with

$$
W_{ \pm}[v(x)]=v_{ \pm}(x) \frac{1}{D_{ \pm}} \partial_{ \pm}, \quad D_{ \pm}=\partial_{ \pm}+g v_{ \pm}(x) .
$$

Apart from the usual QCD Feynman rules, the Feynman rules of the effective action comprise the propagator of the reggeized gluon and an infinite number of so-called induced vertices, which result from the non-local functional Eq. (5). Lowest order vertices and propagators are collected in Fig. 1 (for further details we refer to Ref. [12]). Loop diagrams of the effective action lead to a new type of longitudi- 


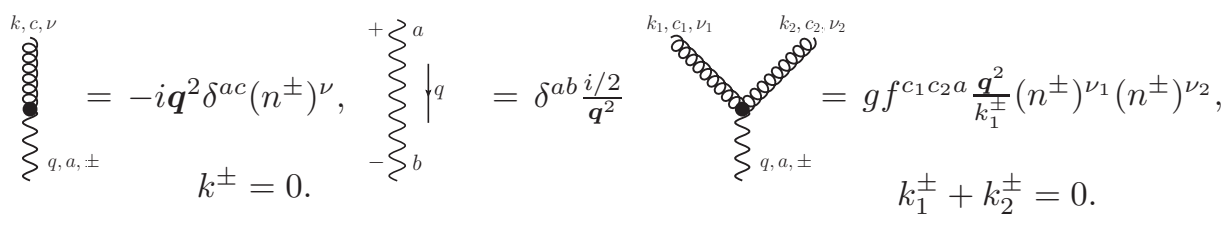

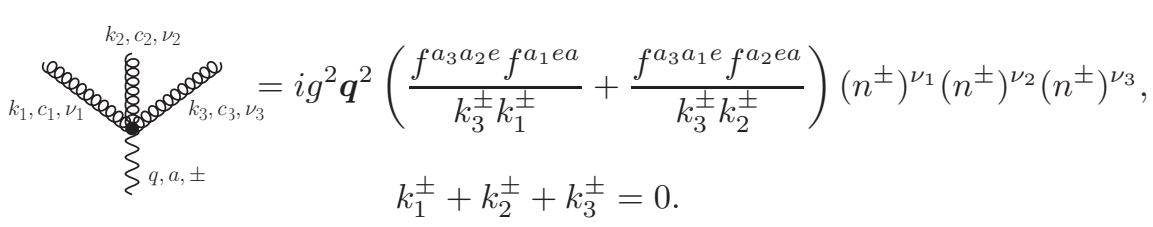

Fig. 1. Feynman rules for the lowest-order effective vertices of the effective action. Wavy lines denote reggeized fields and curly lines gluons.

nal divergence which is not present in conventional quantum corrections to QCD amplitudes. It can be regularized introducing an external parameter $\rho$, evaluated in the limit $\rho \rightarrow \infty$, which deforms the light-like vectors $n^{ \pm}$into

$$
\begin{aligned}
& n^{-} \rightarrow n_{a}=e^{-\rho} n^{+}+n^{-}, \\
& n^{+} \rightarrow n_{b}=n^{+}+e^{-\rho} n^{-},
\end{aligned}
$$

without violating the gauge invariance properties of the induced term Eq. (4). While it is possible to identify $\rho$ with a logarithm in $s$ or the rapidity interval spanned by a certain high energy process, we refrain from such an interpretation and consider in the following $\rho$ as an external parameter, similar to the parameter $\epsilon$ in dimensional regularization in $d=4+2 \epsilon$ dimensions. The evaluation of loop diagrams requires a prescription to circumvent the light-cone singularities in the induced vertices shown in Figs. 1. A suitable prescription which preserves the color structure of unregulated vertices has been derived in Ref. [19], by performing (a) the replacement $D_{ \pm} \rightarrow$ $D_{ \pm} \pm \epsilon$ in Eq. (5) and (b) subsequently projecting out symmetric color structures, order by order in perturbation theory. The resulting pole prescription respects then Bose symmetry of the induced vertices and high energy factorization, for details see Ref. [19]. For the $\mathcal{O}(g)$ vertex, see Fig. 1.c, this corresponds to replacing the denominator $1 / k_{1}^{ \pm}$by a Cauchy principal value. For the $\mathcal{O}\left(g^{2}\right)$ vertex, see Fig. 1.c, the combinations of denominators $1 /\left(k_{3}^{ \pm} k_{1}^{ \pm}\right)$and $1 /\left(k_{3}^{ \pm} k_{2}^{ \pm}\right)$are replaced by functions $g_{2}^{ \pm}(3,2,1)$ and $g_{2}^{ \pm}(3,1,2)$ respectively. It is defined as

$$
g_{2}^{ \pm}(i, j, m)=\left[\frac{1}{\left[k_{i}^{ \pm}\right]\left[k_{m}^{ \pm}\right]}+\frac{\pi^{2}}{3} \delta\left(k_{i}^{ \pm}\right) \delta\left(k_{m}^{ \pm}\right)\right], \quad \frac{1}{[k]} \equiv \frac{1}{2}\left(\frac{1}{k+i 0}+\frac{1}{k-i 0}\right)
$$

with similar functions for the higher order induced vertices. 


\section{The Gluon Regge Trajectory and the Effective Action}

Determination of the gluon trajectory from the effective action requires

- to determine the propagator of the reggeized gluon to the desired order in perturbation theory;

- to renormalize its rapidity divergences.

The gluon Regge trajectory is then identified as the coefficient of the $\rho$ dependent term in the renormalization factor. To derive the 2-loop gluon Regge trajectory we therefore need to determine the 1- and 2-loop self-energies of the reggeized gluon. Within the subtraction procedure proposed in Ref. [9] this requires

- determination of the self-energy of the reggeized gluon from the effective action, with the reggeized gluon treated as a background field;

- subtraction of all disconnected contributions which contain internal reggeized gluon lines.

At 1-loop, all diagrams with internal reggeized gluon lines vanish and no subtraction occurs; the contributing diagrams are

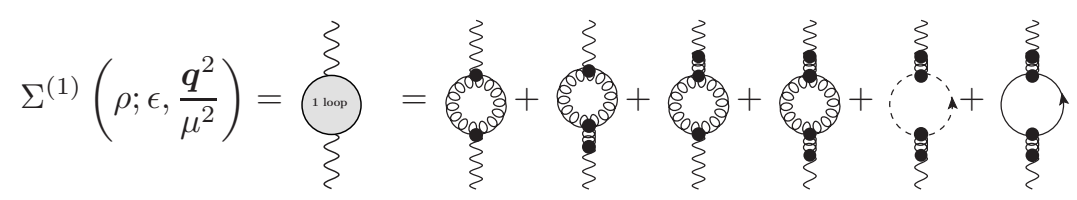

Keeping for $\rho \rightarrow \infty$ the $\mathcal{O}\left(\rho, \rho^{2}\right)$ terms and using the notation

$$
\bar{g}^{2}=\frac{g^{2} N_{c} \Gamma(1-\epsilon)}{(4 \pi)^{2+\epsilon}}, \quad c_{\Gamma}=\frac{\Gamma^{2}(1+\epsilon)}{\Gamma(1+2 \epsilon)},
$$

we have the following result in $d=4+2 \epsilon$ dimensions $^{\mathrm{b}}$ :

$$
\frac{\Sigma^{(1)}\left(\rho ; \epsilon, \frac{\boldsymbol{q}^{2}}{\mu^{2}}\right)}{\left(-2 i \boldsymbol{q}^{2}\right)}=\bar{g}^{2} c_{\Gamma}\left(\frac{\boldsymbol{q}^{2}}{\mu^{2}}\right)^{\epsilon}\left\{\frac{i \pi-2 \rho}{\epsilon}-\frac{5+3 \epsilon-\frac{n_{f}}{N_{c}}(2+2 \epsilon)}{(1+2 \epsilon)(3+2 \epsilon) \epsilon}\right\} .
$$

To determine the 2-loop self energy it is on the other hand needed to subtract disconnected diagrams, whereas diagrams with multiple internal reggeized gluons

\footnotetext{
${ }^{\mathrm{b}}$ In the original result presented in Ref. [9] and reproduced in Ref. [11, 18] a finite result for the second and third diagram has been erroneously included. This has been corrected in the result presented here.
} 
can be shown to yield a zero result. Schematically one has

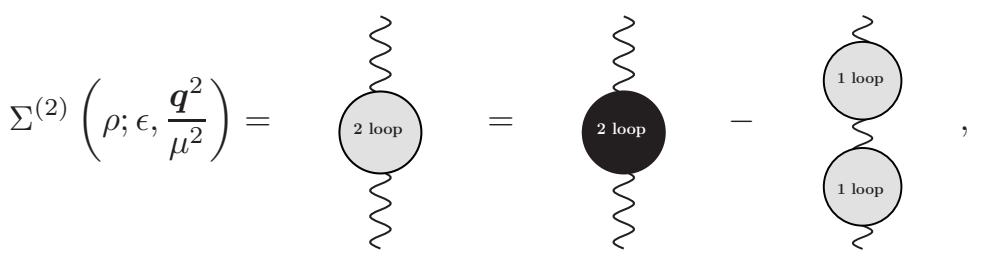

where the black blob denotes the unsubtracted 2-loop reggeized gluon self-energy, which is obtained through the direct application of the Feynman rules of the effective action, with the reggeized gluon itself treated as a background field. The emerging set of Feynman integrals can be reduced to seven master integrals which have been evaluated in Ref. [12] using the Mellin-Barnes representation technique and expansion in $\rho$ using the Mathematica package MBasymptotics.m [23]; the singularities structure in $\epsilon$ was subsequently resolved using the Mathematica packages MB.m [21] and MBresolve.m [22]. For details we refer to Ref. $[11,12]$. The result for $n_{f}$ flavor reads

$$
\begin{aligned}
& \Sigma^{(2)}\left(\rho, \frac{\boldsymbol{q}^{2}}{\mu^{2}}\right)=\left(-2 i \boldsymbol{q}^{2}\right) \frac{g^{4} N_{c}^{2}}{(4 \pi)^{4}}\left\{-\left[\frac{2}{\epsilon^{2}}+\frac{4(1-\Xi)}{\epsilon}+4(1-\Xi)^{2}-\frac{\pi^{2}}{3}\right] \rho^{2}\right. \\
& \left.\left.+\left[\frac{1}{3 \epsilon^{2}}+\frac{1}{9 \epsilon}+\frac{\pi^{2}}{3 \epsilon}-\frac{2 \Xi}{3 \epsilon}+\frac{\pi^{2}(11-12 \Xi)}{18}+\frac{16}{27}-\frac{2}{9} \Xi+\frac{2}{3} \Xi^{2}-2 \zeta(3)\right)\right] \rho\right\} \\
& +\frac{n_{f}}{N_{c}}\left(\frac{2}{3 \epsilon}+\frac{n f(6-36 \Xi)}{27 \epsilon}+\frac{32-3 \pi^{2}-12 \Xi+36 \Xi^{2}}{27}\right)+\mathcal{O}(\epsilon)+\mathcal{O}\left(\rho^{0}\right) .
\end{aligned}
$$

with $\Xi=1-\gamma_{E}-\ln \boldsymbol{q}^{2} /\left(4 \pi \mu^{2}\right)$. The (bare) two-loop reggeized gluon propagators is then in term of 1- and 2-loop self energies given by

$$
G\left(\rho ; \epsilon, \boldsymbol{q}^{2}, \mu^{2}\right)=\frac{i / 2}{\boldsymbol{q}^{2}}\left\{1+\frac{i / 2}{\boldsymbol{q}^{2}} \Sigma\left(\rho ; \epsilon, \frac{\boldsymbol{q}^{2}}{\mu^{2}}\right)+\left[\frac{i / 2}{\boldsymbol{q}^{2}} \Sigma\left(\rho ; \epsilon, \frac{\boldsymbol{q}^{2}}{\mu^{2}}\right)\right]^{2}+\ldots\right\}
$$

with

$$
\Sigma\left(\rho ; \epsilon, \frac{\boldsymbol{q}^{2}}{\mu^{2}}\right)=\Sigma^{(1)}\left(\rho ; \epsilon, \frac{\boldsymbol{q}^{2}}{\mu^{2}}\right)+\Sigma^{(2)}\left(\rho ; \epsilon, \frac{\boldsymbol{q}^{2}}{\mu^{2}}\right)+\ldots
$$

where the dots indicate higher order terms. Apparently Eq. (10) is divergent in the limit $\rho \rightarrow \infty$. In Ref. $[9,10]$ it has been demonstrated by explicit calculations that these divergences cancel at one-loop level, for both quark-quark and gluon-gluon scattering amplitudes, against divergences in the couplings of the reggeized gluon to external particles. The entire one-loop amplitude is then found to be free of any high energy singularity in $\rho$. High energy factorization then suggests that such a cancellation holds also beyond one loop. Starting from this assumption, it is possible 
to define a renormalized reggeized gluon propagator

$$
G^{\mathrm{R}}\left(M^{+}, M^{-} ; \epsilon, \boldsymbol{q}^{2}, \mu^{2}\right)=\frac{G\left(\rho ; \epsilon, \boldsymbol{q}^{2}, \mu^{2}\right)}{Z^{+}\left(\frac{M^{+}}{\sqrt{\boldsymbol{q}^{2}}}, \rho ; \epsilon, \frac{\boldsymbol{q}^{2}}{\mu^{2}}\right) Z^{-}\left(\frac{M^{-}}{\sqrt{\boldsymbol{q}^{2}}}, \rho ; \epsilon, \frac{\boldsymbol{q}^{2}}{\mu^{2}}\right)},
$$

where the renormalization factors need to cancel against corresponding renormalization factors associated with the vertex to which the reggeized gluon couples with 'plus' $\left(Z^{+}\right)$and 'minus' $\left(Z^{-}\right)$polarization. For explicit examples we refer the reader to Ref. $[10,11]$. In their most general form these renormalization factors are parametrized as

$$
Z^{ \pm}\left(\frac{M^{ \pm}}{\sqrt{\boldsymbol{q}^{2}}}, \rho ; \epsilon, \frac{\boldsymbol{q}^{2}}{\mu^{2}}\right)=\exp \left[\left(\frac{\rho}{2}-\ln \frac{M^{ \pm}}{\sqrt{\boldsymbol{q}^{2}}}\right) \omega\left(\epsilon, \frac{\boldsymbol{q}^{2}}{\mu^{2}}\right)+f^{ \pm}\left(\epsilon, \frac{\boldsymbol{q}^{2}}{\mu^{2}}\right)\right] .
$$

The coefficient of the $\rho$-divergent term defines the gluon Regge trajectory $\omega\left(\epsilon, \boldsymbol{q}^{2}\right)$, with the the following perturbative expansion

$$
\omega\left(\epsilon, \frac{\boldsymbol{q}^{2}}{\mu^{2}}\right)=\omega^{(1)}\left(\epsilon, \frac{\boldsymbol{q}^{2}}{\mu^{2}}\right)+\omega^{(2)}\left(\epsilon, \frac{\boldsymbol{q}^{2}}{\mu^{2}}\right)+\ldots
$$

It is to be determined by the requirement that the renormalized reggeized gluon propagator must, at each loop order, be free of $\rho$ divergences. At one loop one obtains

$$
\omega^{(1)}\left(\epsilon, \frac{\boldsymbol{q}^{2}}{\mu^{2}}\right)=-\frac{2 \bar{g}^{2} \Gamma^{2}(1+\epsilon)}{\Gamma(1+2 \epsilon) \epsilon}\left(\frac{\boldsymbol{q}^{2}}{\mu^{2}}\right)^{\epsilon} .
$$

The function $f^{ \pm}\left(\epsilon, \boldsymbol{q}^{2}\right)$ parametrizes finite contributions and is, in principle, arbitrary. While symmetry of the scattering amplitude requires $f^{+}=f^{-}=f$, Regge theory suggests fixing it in such that terms which are not enhanced in $\rho$ are entirely transferred from the reggeized gluon propagators to the vertices, to which the reggeized gluon couples. With the perturbative expansion

$$
f\left(\epsilon, \frac{\boldsymbol{q}^{2}}{\mu^{2}}\right)=f^{(1)}\left(\epsilon, \frac{\boldsymbol{q}^{2}}{\mu^{2}}\right)+f^{(2)}\left(\epsilon, \frac{\boldsymbol{q}^{2}}{\mu^{2}}\right) \ldots
$$

we obtain from Eq. (10)

$$
f^{(1)}\left(\epsilon, \frac{\boldsymbol{q}^{2}}{\mu^{2}}\right)=\frac{\bar{g}^{2} \Gamma^{2}(1+\epsilon)}{\Gamma(1+2 \epsilon)}\left(\frac{\boldsymbol{q}^{2}}{\mu^{2}}\right)^{\epsilon} \frac{(-1)}{(1+2 \epsilon) 2 \epsilon}\left[\frac{5+3 \epsilon}{3+2 \epsilon}-\frac{n_{f}}{N_{c}}\left(\frac{2+2 \epsilon}{3+2 \epsilon}\right)\right] .
$$

The scales $M^{+}$and $M^{-}$are arbitrary; their role is analogous to the renormalization scale in UV renormalization and the factorization scale in collinear factorization. They are naturally chosen to be of the order of magnitude of the corresponding light-cone momenta of scattering particles to which the reggeized gluon couples. For the 2-loop gluon Regge trajectory we obtain the following relation

$$
\omega^{(2)}\left(\epsilon, \frac{\boldsymbol{q}^{2}}{\mu^{2}}\right)=\lim _{\rho \rightarrow \infty} \frac{1}{\rho}\left[\frac{\Sigma^{(2)}}{\left(-2 i \boldsymbol{q}^{2}\right)}+\frac{\rho^{2}}{2}\left(\omega^{(1)}\right)^{2}+2 \rho f^{(1)} \omega^{(1)}\right],
$$

where we omitted at the right hand side the dependencies on $\epsilon$ and $\boldsymbol{q}^{2} / \mu^{2}$ and expanded $\Sigma^{(1)}$ in terms of the functions $\omega^{(1)}$ and $f^{(1)}$. We stress that this is a 
non-trivial definition and that it is not clear a priori whether the right hand side even exists due to the presence of the second term, linear in $\rho$. Confirmation of this relation provides therefore an important non-trivial check on the validity of our formalism. Inserting our result for the 2-loop reggeized gluon self-energy into Eq. (21) we obtain

$$
\omega^{(2)}\left(\boldsymbol{q}^{2}\right)=\frac{\left(\omega^{(1)}\left(\boldsymbol{q}^{2}\right)\right)^{2}}{4}\left[\frac{11}{3}-\frac{2 n_{f}}{3 N_{c}}+\left(\frac{\pi^{2}}{3}-\frac{67}{9}\right) \epsilon+\left(\frac{404}{27}-2 \zeta(3)\right) \epsilon^{2}\right] .
$$

which is in complete agreement with the results in the literature. ${ }^{13}$

\section{Conclusions}

In this contribution the central steps of the derivation of the two-loop gluon Regge trajectory from Lipatov's effective action have been presented. They consist of determination of the reggeized gluon propagator to the desired order in perturbation theory and subsequent renormalization of high energy divergences. The coefficient of the high energy divergence is then identified as the gluon Regge trajectory. Determination of the reggeized gluon propagator requires on the other hand evaluation of corresponding effective action diagrams, combined with a subtraction mechanism. The final result is in precise agreement with earlier results present in the literature and thus serves to further validate the effective action and our proposed computational framework.

\section{Acknowledgements}

We thank J. Bartels, V. Fadin and L. Lipatov for constant support for many years. We acknowledge partial support by the Research Executive Agency (REA) of the European Union under the Grant Agreement number PITN-GA-2010-264564 (LHCPhenoNet), the Comunidad de Madrid through Proyecto HEPHACOS ESP1473, by MICINN (FPA2010-17747), by the Spanish Government and EU ERDF funds (grants FPA2007-60323, FPA2011-23778 and CSD2007- 00042 Consolider Project CPAN) and by GV (PROMETEUII/2013/007). G.C. acknowledges support from Marie Curie Actions (PIEF-GA-2011-298582). M.H. acknowledges support from the U.S. Department of Energy under contract number DE-AC02-98CH10886 and a "BNL Laboratory Directed Research and Development" grant (LDRD 12$034)$.

\section{References}

1. L. N. Lipatov, Sov. J. Nucl. Phys. 23, 338 (1976); E. A. Kuraev, L. N. Lipatov, V. S. Fadin, Phys. Lett. B 60, 50 (1975), Sov. Phys. JETP 44, 443 (1976); Sov. Phys. JETP 45, 199 (1977); Ia. Ia. Balitsky, L. N. Lipatov, Sov. J. Nucl. Phys. 28, $822(1978)$.

2. V. S. Fadin, L. N. Lipatov, Phys. Lett. B 429, 127 (1998); M. Ciafaloni, G. Camici, Phys. Lett. B 430, 349 (1998). 
3. J. Ellis, H. Kowalski and D. A. Ross, Phys. Lett. B 668, 51 (1998); H. Kowalski, L. N. Lipatov, D. A. Ross and G. Watt, Eur. Phys. J. C 70 , 983( 2010).

4. M. Hentschinski, A. Sabio Vera and C. Salas, Phys. Rev. Lett. 110, 041601 (2013); Phys. Rev. D 87, 076005 (2013).

5. K. Dusling and R. Venugopalan, Phys, Rev. D 87, 051502 (2013).

6. H. Jung, S. Baranov, M. Deak, A. Grebenyuk, F. Hautmann, M. Hentschinski, A. Knutsson and M. Kramer et al., Eur. Phys. J. C 70, 1237 (2010); H. Jung and F. Hautmann, arXiv:1206.1796 [hep-ph]; F. Hautmann, M. Hentschinski and H. Jung, Nucl. Phys. B 865, 54 (2012); M. A. Nefedov, V. A. Saleev and A. V. Shipilova, Phys. Rev. D 87, 094030 (2013).

7. L. N. Lipatov, Nucl. Phys. B452, 369 (1995); Phys. Rept. 286, 131 (1997).

8. E. N. Antonov, L. N. Lipatov, E. A. Kuraev and I. O. Cherednikov, Nucl. Phys. B 721, 111 (2005).

9. M. Hentschinski and A. Sabio Vera, Phys. Rev. D 85, 056006 (2012)

10. G. Chachamis, M. Hentschinski, J. D. Madrigal and A. Sabio Vera, Phys. Rev. D 87, 076009 (2012).

11. G. Chachamis, M. Hentschinski, J. D. Madrigal and A. Sabio Vera, Nucl. Phys. B 861, 133 (2012).

12. G. Chachamis, M. Hentschinski, J. D. Madrigal and A. Sabio Vera, arXiv:1307.2591 [hep-ph] (2013).

13. V. S. Fadin, R. Fiore, M. I. Kotsky, Phys. Lett. B387, 593 (1996).

14. V. S. Fadin, R. Fiore and A. Quartarolo, Phys. Rev. D 53, 2729 (1996); M. I. Kotsky and V. S. Fadin, Phys. Atom. Nucl. 59, 1035 (1996) [Yad. Fiz. 59N6, 1080 (1996)]; V. S. Fadin, M. I. Kotsky and R. Fiore, Phys. Lett. B 359, 181 (1995).

15. J. Blümlein, V. Ravindran and W. L. van Neerven, Phys. Rev. D 58, 091502 (1998).

16. I. A. Korchemskaya and G. P. Korchemsky, Phys. Lett. B 387, 346 (1996).

17. V. Del Duca and E. W. N. Glover, JHEP 0110, 035 (2001).

18. G. Chachamis, M. Hentschinski, J. D. Madrigal and A. Sabio Vera, arXiv:1211.2050 [hep-ph], to appear in Phys. Part. Nucl..

19. M. Hentschinski, Nucl. Phys. B 859, 129 (2012)

20. V. S. Fadin, "BFKL news," hep-ph/9807528 (1998); B. L. Ioffe, V. S. Fadin and L. N. Lipatov, "Quantum chromodynamics: Perturbative and nonperturbative aspects," Cambridge monographs on Particle Physics, Nuclear Physics and Cosmology (No. 30) (2010).

21. M. Czakon, Comput. Phys. Commun. 175, 559 (2006).

22. A. V. Smirnov and V. A. Smirnov, Eur. Phys. J. C 62, 445 (2009).

23. M. Czakon, MBasymptotics.m, http://projects.hepforge.org/mbtools/. 